\title{
Modelling and quantifying the effect of heterogeneity in soil physical conditions on fungal growth
}

\author{
R. Pajor, R. Falconer, S. Hapca, and W. Otten \\ The SIMBIOS Centre, University of Abertay Dundee, Kydd Building, Dundee, DD1 1HG, UK \\ Received: 9 April 2010 - Published in Biogeosciences Discuss.: 12 May 2010 \\ Revised: 31 October 2010 - Accepted: 1 November 2010 - Published: 18 November 2010
}

\begin{abstract}
Despite the importance of fungi in soil ecosystem services, a theoretical framework that links soil management strategies with fungal ecology is still lacking. One of the key challenges is to understand how the complex geometrical shape of pores in soil affects fungal spread and species interaction. Progress in this area has long been hampered by a lack of experimental techniques for quantification. In this paper we use X-ray computed tomography to quantify and characterize the pore geometry at microscopic scales $(30 \mu \mathrm{m})$ that are relevant for fungal spread in soil. We analysed the pore geometry for replicated samples with bulk-densities ranging from $1.2-1.6 \mathrm{~g} / \mathrm{cm}^{3}$. The bulk-density of soils significantly affected the total volume, mean pore diameter and connectivity of the pore volume. A previously described fungal growth model comprising a minimal set of physiological processes required to produce a range of phenotypic responses was used to analyse the effect of these geometric descriptors on fungal invasion, and we showed that the degree and rate of fungal invasion was affected mainly by pore volume and pore connectivity. The presented experimental and theoretical framework is a significant first step towards understanding how environmental change and soil management impact on fungal diversity in soils.
\end{abstract}

\section{Introduction}

The pivotal role of fungi in ecosystem functioning is now widely accepted, and soil management strategies that support fungal diversity are to be encouraged. Fungi are ubiquitous microorganisms in soil $(0.8-16 \mathrm{~km}$ of hyphae per $1 \mathrm{~g}$ of soil

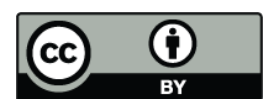

Correspondence to: W. Otten

(w.otten@abertay.ac.uk)
(Young et al., 2008; Finlay, 2006)) and they have a significant influence on aggregation and stabilisation of soil particles (Bossuyt et al., 2001; Tisdall, 1991), nutrient and carbon dynamics (Taylor et al., 2009), and many soil-borne diseases (Otten et al., 2004). Their unique mycelial form of growth makes them particularly suited for exploration of very heterogeneous environments such as soil (Boswell 2007, Otten and Gilligan, 1998). Unlike for bacteria, colonisation of soil by fungi is not limited to water-filled volumes, and they can readily overcome locally less suitable growth conditions and patchy nutrient distributions. However, very little work has looked at how fungi colonize soil and how microscopic heterogeneity affects the colony morphology, and a theoretical framework of species interaction in a 3-D heterogeneous soil environment is still lacking.

One of the challenges in studying species interactions in soil is that soils are heterogeneous at spatial scales ranging from the micrometer (reflecting the pores within which microbial interactions take place) to kilometres. Whereas the heterogeneity of soils at larger scales has received considerable attention (Lark, 2005), the microscopic heterogeneity has been largely ignored. The size of bacterial cells is less than $10 \mu \mathrm{m}$, fungal diameters range roughly from $1-50 \mu \mathrm{m}$ and fungal colonies are of the order of $\mathrm{cm}$, hence even at the scales of micro-organisms we already bridge $10^{3}$ orders of magnitude (Finlay, 2006). The heterogeneity of soil structure at the micro scale (pore scale) controls the flow of water, the availability of nutrients, and the diffusion of oxygen to micro-organisms (Young and Crawford, 2004). At those scales, soil structure creates spatially separate niches for various microorganisms (Or et al., 2007), and preferential pathways through which they can move either autonomously or via convective transport with water.

Published by Copernicus Publications on behalf of the European Geosciences Union. 
The opacity of soils and a lack of non-invasive quantitative techniques to study growth dynamics of fungi in situ make it difficult to understand how the microscopic heterogeneity of soils affects fungal dynamics and contributes to biodiversity. Conventional soil physical techniques to characterise soil structure have concentrated on bulk physical parameters where we quantify for example aggregate size distributions (after we exerted physical forces upon the soil) or bulkdensity. While we can derive overall porosity values from these measures, they tell us very little about the geometry and connectivity of the pore space. Other methods for analysis of the pore space are based on destructive sampling such as thin sectioning (Harris et al., 2003; Nunan et al., 2001), or on information of pore size distribution derived from water retention curves (Dane et al., 2002). Neither of these methods accurately account for the 3-D structure and connectivity of the pore space. Only recently, the development of techniques such as X-ray micro-tomography allow for quantification and visualisation of the internal soil structure without destroying the sample. A soil sampling ring with $4 \mathrm{~cm}$ diameter can be readily scanned within $60 \mathrm{~min}$ at a resolution of approximately $30 \mu \mathrm{m}$. For smaller samples resolutions of $<1 \mu \mathrm{m}$ can be obtained. However, current capabilities of Xray micro-tomography systems still do not allow us to visualise and quantify the dynamics of fungi in soils. Neither are there currently other techniques that can quantify the spatial distribution of fungi within a 3-D heterogeneous structure at microscopic scales.

The use of mathematical modelling offers a way forward. With models we can study precisely the effect of the pore geometry on fungal growth, as conditions that are correlated in experimental systems can be controlled separately. There are a number of fungal growth models which consider fungal growth dynamics at different spatial scales: the colony (cm's) or the hyphal scale $(\mu \mathrm{m})$. The most recent models merge both scales which is important to predict colony dynamics from interactions between hyphae and the environment. Most models are based on earlier work by Edelstein (1982) and Edelstein and Segel (1983) who considered fungal spread at colony scales. Bosswel et al. (2002) extended these models by including directional growth and bidirectional translocation mechanisms. Stacey et al. (2001) developed a model to scale-up from hyphae to the colony level. This work was used to investigate transmission rates of plant pathogens. Vectorial-based models (Meskauskas et al., 2004) moved analysis from 2-D to 3-D with the possibility to model fruiting bodies. An ecologically important characteristic of fungi is that they can spread via hyphal growth and translocate nutrients over several cm's within a colony, making them particularly adapt for heterogeneous environments such as soil. Under nutrient pore conditions, biomass can be relocated within a colony and support further fungal growth. However most of these models do not include the ability of fungi to reuse their own biomass (hereafter referred to as recycling) hence they are less suitable for heterogeneous envi- ronments. The model we used in this work is a fungal growth model developed by Falconer (Falconer et al., 2005), and described below. Uniquely, this model can model fungal spread in 3-D, and can be combined with the X-ray CT data that describe the pore geometry. This is the first time that this model will be applied to a range of pore geometries that result from different bulk-densities.

The main aim of this work is to quantify and visualise the effect of the internal structure of soil on fungal growth dynamics and colonization efficiency in 3-D. First we will investigate how the pore geometry of microcosms prepared at a range of soil densities is affected at microscopic scales, and then we will use theoretical modelling to test which of these descriptors of the pore geometry affect fungal colonization. We first conduct the simulations with a high $\mathrm{C}$ content in the soil sample to ensure fungal growth is not constrained, and then test if the same response of fungal spread to pore geometry is found in C-limited conditions.

\section{Materials and methods}

\subsection{Preparation of soil microcosms}

We used a sandy loam soil (organic matter 2.6\%; sand, $71 \%$; silt, $19 \%$; clay, $10 \%$; pH 6.2) sampled from an experimental site (Bullion field) of SCRI (Scottish Crop Research Institute UK). The soil was air-dried and sieved to obtain aggregates sized $1-2 \mathrm{~mm}$. The soil was sterilized by double autoclaving (1h cycles with $48 \mathrm{~h}$ intervals) prior to packing. Soil was packed into the PVC rings at densities of $1.2 \mathrm{~g} / \mathrm{cm}^{3}(n=3)$, $1.3 \mathrm{~g} / \mathrm{cm}^{3}(n=2), 1.4 \mathrm{~g} / \mathrm{cm}^{3}(n=3), 1.5 \mathrm{~g} / \mathrm{cm}^{3}(n=4)$ and $1.6 \mathrm{~g} / \mathrm{cm}^{3}(n=2)$. These soils were used in a previous study where the invasion of fungi into soil was investigated in thin sections from these samples (Harris et al., 2003), for which the samples were resin impregnated. The aim was to produce samples that differ in physical characteristics of pore-space to test its effect on fungal colonization.

\subsection{Quantification of soil structure}

A Metris X-Tek X-ray micro-tomography system was used for quantification and visualisation of the inner pore space of the soil microcosms. All soil microcosms were scanned at $160 \mathrm{kV}, 201 \mu \mathrm{A}$ and 3003 angular projection, 4 frames per second and a $0.1 \mathrm{~mm}$ Al filter. Radiographs were reconstructed into a 3-D volume using CT-Pro (Nikon), imported into VGStudiomax (http://www.volumegraphics.com/), and converted into 8-bit binary TIFF image stacks with voxelthick slices. All soil samples were scanned and reconstructed into 3-D volumes at a resolution of $30 \mu \mathrm{m}$ (voxel size). The reconstructed volumes were cropped to obtain equally sized volumes for all samples of $300 \times 300 \times 300$ voxels $(9 \mathrm{~mm} \times 9 \mathrm{~mm} \times 9 \mathrm{~mm})$. Because the scanned, large volumes were not uniform these samples were not cropped around 
a fixed midpoint but cropped to avoid areas with ring artefacts and noise related to scanning, and predominantly occurring around the edges of each sample. A single global threshold value was set for each of these samples. Binary data sets were created by thresholding the greyscale image stacks in ImageJ. The choice of threshold value was based on the histogram region corresponding with the pore-solid interface, taking into account variation of grey scale values in pores of different shapes and sizes, and averaging over 5 randomly selected slices per sample using Image $\mathbf{J}$ (Image $\mathrm{J}$, http://rsb.info.nih.gov/ij/). In a study comparing different thresholding methods it was shown that this methodology predicted porosity close to the mean value of all tested methods and agreed well with the overall porosity of the bulk soil sample (Baveye et al., 2010). To allow for analysis of the effect of pore geometry on fungal growth, the thresholded (binary) datasets were further divided in a consistent way into eight subsamples (pseudo replicates) with dimensions of $128 \times 128 \times 128$ voxels (see Fig. 1). This division was necessary due to computational limitations of the fungal growth model in a 3-D space (see below). In this work replicates of each treatment (density, $n=2-4$ ) will be referred to as the samples, and datasets sized $128^{3}$ will be called subsamples.

We quantified the following physical properties of the subsamples (Deurer et al., 2009):

- porosity- the total number of voxels defined as pores divided by total volume of the sample. This represents the maximum volume in a soil sample within which fungi can potentially spread.

- pore space connectivity - A voxel is considered to belong to the same cluster if one of the six directly neighbouring voxels was identified as pore space. We quantified the number of separate pore clusters and the percentage of the pore volume belonging to each of those clusters. For our analysis we focus on the percentage of the pore volume belonging to the largest cluster, as this was the only connected pore volume large enough to spread over the entire width of the soil sample.

- pore sizes distribution - We calculated the distribution of pore radii by simulating a growing sphere at every voxel of pore space till it reached a voxel with solid phase and we plotted the distribution of the radii of the spheres.

\subsection{Fungal growth model}

Fungal growth was modelled using the framework developed by Falconer et al. (2005). This model is parsimonious in construction and reduces the biological complexity capturing the minimal set of physiological processes required to reproduce observed ranges in phenotypic responses (Falconer et al., 2005). It was shown that the model can capture fungal

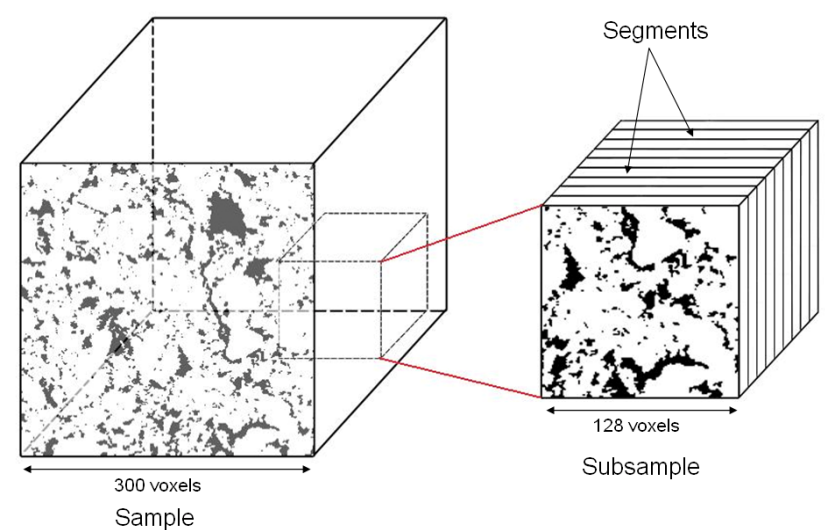

Fig. 1. 3-D spatial arrangement of the data structure. Treatments were compared by comparing physical properties for cubed samples. Within each sample, subsamples were selected, which were divided in segments to enable quantification of fungal invasion. Fungal invasion was initiated from the first segment in each subsample.

growth dynamics in homogeneous as well as in nutritionally heterogeneous environments (Falconer et al., 2007). The model is based on five physiological processes: uptake, redistribution of biomass, remobilisation of biomass, inhibitor production, and growth. Spread of biomass in the model is effectively described by a diffusive process. All of physiological processes are known to be important for vegetative growth of fungi but have not been collectively included in any other modelling framework. For a detailed explanation of the model the reader is referred to Falconer et al. (2005). The model can simulate growth in a 3-D pore space. As the objective of this study is to analyse the effect of pore geometry, we used parameters for one single fungal species only. In previous work the fungal trait set for effective invasion of heterogeneous environments was identified (Falconer et al., 2008). Simplified assumptions were made with respect to the nutritional heterogeneity of the soil environment: we assumed Carbon to be homogeneously distributed throughout the pore volume. We analysed the effect of a high (100, C units per voxel) and low (10, C units per voxel) carbon content on fungal growth dynamics to test if our results were dominated by the availability of resources. At the start of the simulation, fungal biomass was placed only in a unit-thick voxel vertical plane (Fig. 1). Fungal spread was initiated from this plane and followed throughout the sample. The simulations were terminated when a threshold value of total biomass $\left(10^{-6}\right)$ reached the opposite edge of the subvolume (break through time).

\subsection{Interpretation of output from the model}

To enable for comparison of fungal invasion among treatments we captured the dynamics and spatial distribution of fungal invasion by dividing each subsample into segments 
that were perpendicular to the direction of fungal growth, and parallel to the plane of inoculation (Fig. 1). Following our analysis for the physical properties, we quantified the following characteristics:

- Biomass per segment: this quantifies at each time step the amount of biomass per segment at specified distances from the site of inoculation, as a measure of the ability of fungi to invade the soil structure.

- Fraction of pore volume occupied by fungal biomass here we combine the data on the porosity within each segment with the biomass per segment to calculate for each time-step the fraction of pores that are filled with biomass. This measure enables characterization of the efficiency at which the pore volume is colonised by fungi.

\subsection{Statistical analysis}

To test for the effect of the bulk density on soil porosity, medium pore size and measures of connectivity, a nested ANOVA model was used with bulk density as fixed factor (with levels 1.2, 1.3, 1.4, 1.5, 1.6) and the samples as nested factors within the different bulk density levels. Bonferroni post-hoc pair wise comparison tests were carried out to determine significant differences among means.

We used a Generalized Estimation Equations (GEEs) model with normal errors and first order autoregressive correlation structure to test for an effect of bulk-density and distance from the site of inoculation on fungal biomass densities within each subsample. The variables bulk density (with five levels), distance (with nine levels, (segments) corresponding to the distance from the inoculation point), and sub-samples that were nested with the different bulk density levels were used as explanatory variables in the model. More specifically, bulk density was introduced as a between subjects factor, while distance was treated either as within subject covariate or as a factor, as indicated by the Quasi Likelihood under Independence model selection criterion (QIC). An interaction term between factors bulk density and distance was also accommodated in the model and Bonferroni post-hoc pair wise comparison tests were carried out to determine significant differences among means of the different factor levels at a significant level of 0.05 . All the statistical analyses were carried out in SPSS v.17 (Hardin and Hilbe, 2003).

\section{Results}

\subsection{Effect of density on physical properties}

The 3-D geometry of the pore space was substantially affected by the density at which the samples were packed (Fig. 2). Visual examination of the pore volumes in 3-D showed that the total pore space was less in the more densely

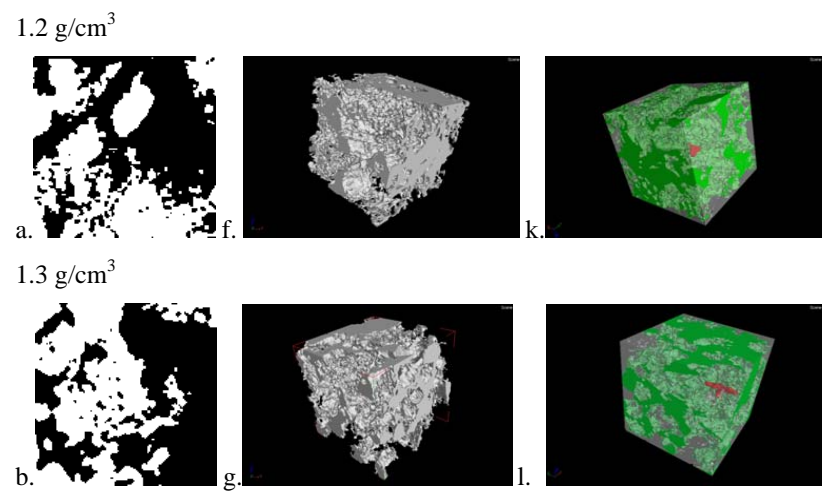

$1.4 \mathrm{~g} / \mathrm{cm}^{3}$
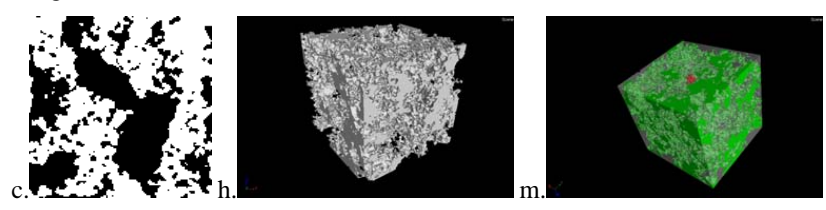

$1.5 \mathrm{~g} / \mathrm{cm}^{3}$
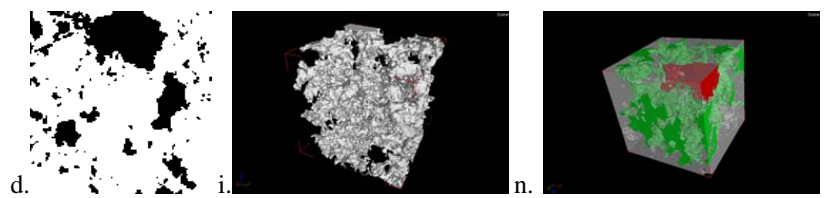

$1.6 \mathrm{~g} / \mathrm{cm}^{3}$
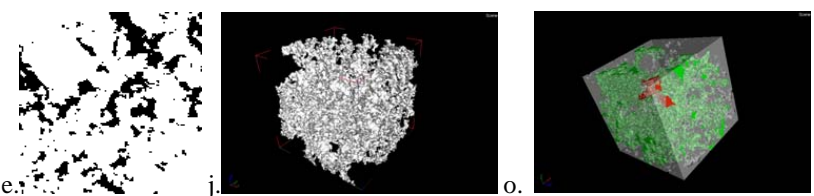

Fig. 2. Pore space visualisation - a-e: thresholded 2-D slices of subsamples (white-solid, black-pore), $\mathbf{f}-\mathbf{j}$ : whole pore space in 3-D as visualized with $\mathrm{X}$-ray $\mathrm{CT}, \mathbf{k - \mathbf { 0 }}$ ) 3-D view of subsample with the largest connected pore (green), the second largest connected pore (red) and the remaining pore space (bright gray). One representative examples is shown for each of the density treatments (1.2-1.6).

packed soil. Whereas in the 2-D transects pores within the pore volume appeared to be disconnected, this was no longer the case when the pore volume was examined in 3-D. In 3$\mathrm{D}$ the majority of the pores was connected and belonged to a single large cluster. In addition, for soil packed at higher densities the pore volume appeared to be connected via smaller valleys. As can be seen from Fig. 2 (k-o) the largest connected cluster was in contact with all sides which in principle will allow for spread of fungi through the soil sample.

Porosity, which is the volume through which fungal growth was modelled, was calculated for each of the subsamples. In addition, porosity was also calculated for segments within the subsamples (see also Fig. 1); the segments represent the smaller scale heterogeneity within each sample. No significant interaction between the different treatment densities and segments was found $(p=0.269)$. The mean porosity 
Table 1. Mean and standard error estimates of bulk physical characteristics for soil microcosms.

\begin{tabular}{ccccccc}
\hline $\begin{array}{c}\text { Sample } \\
\text { density }\left[\mathrm{g} / \mathrm{cm}^{3}\right]\end{array}$ & $\begin{array}{c}\text { Mean } \\
\text { porosity }\end{array}$ & $\begin{array}{c}\text { Standard } \\
\text { error }\end{array}$ & $\begin{array}{c}\text { Mean } \\
\text { connectivity [\%] }\end{array}$ & $\begin{array}{c}\text { Standard } \\
\text { error }\end{array}$ & $\begin{array}{c}\text { Median of } \\
\text { pore size }\end{array}$ & $\begin{array}{c}\text { Standard } \\
\text { error }\end{array}$ \\
\hline 1.2 & 0.34 & 0.02 & 96.5 & 0.5 & 370 & 23 \\
1.3 & 0.38 & 0.02 & 97.0 & 0.3 & 375 & 24 \\
1.4 & 0.31 & 0.01 & 97.0 & 0.4 & 348 & 23 \\
1.5 & 0.26 & 0.01 & 95.1 & 0.2 & 334 & 11 \\
1.6 & 0.21 & 0.01 & 90.0 & 0.6 & 309 & 13 \\
\hline
\end{tabular}
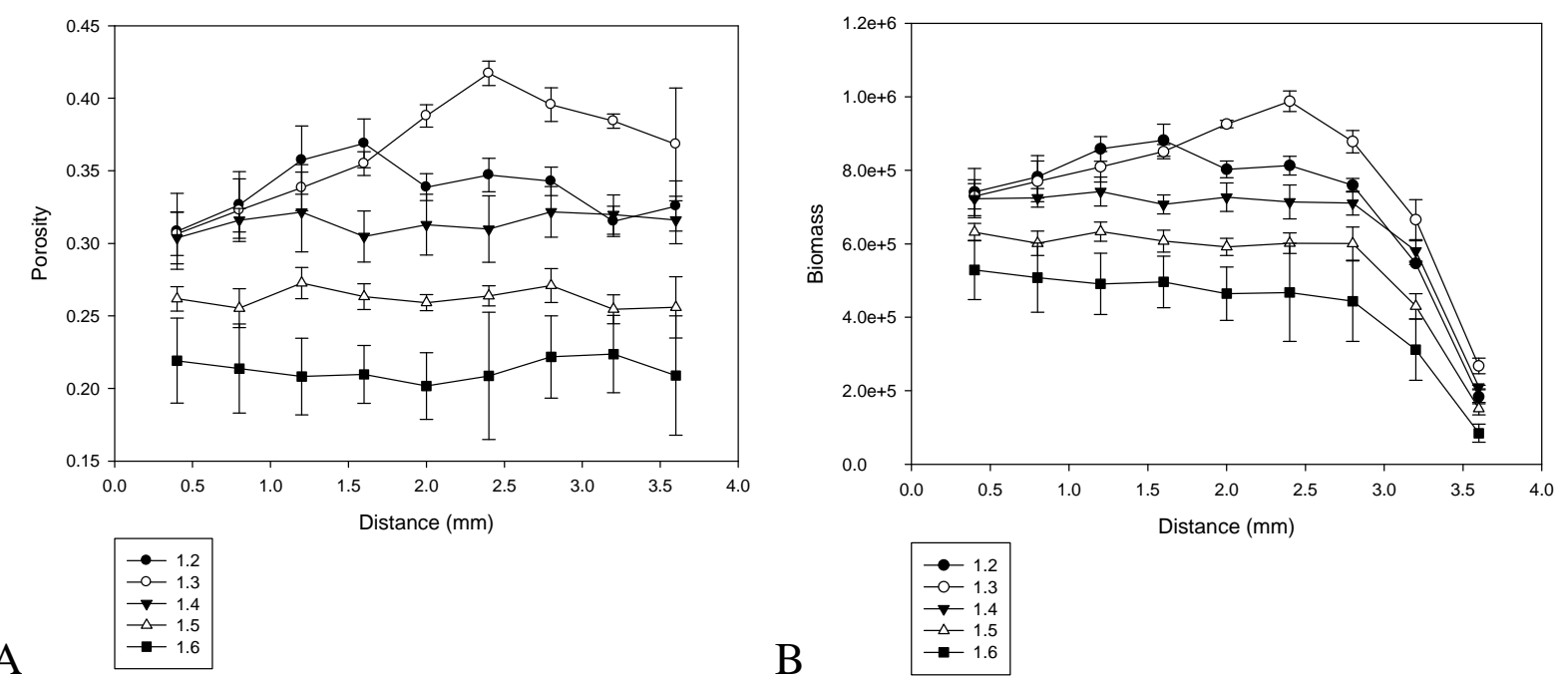

A

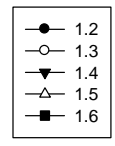

$\mathrm{B}$

Fig. 3. Mean changes of porosity with distance from the site of inoculation (A), and the simulated biomass distribution in each segment (B), for soils at bulk-densities of $1.2,1.3,1.4,1.5$, and $1.6 \mathrm{~g} / \mathrm{cm}^{3}$.

was very strongly affected by the density $(p<0.001)$ ranging from 0.38 for density $1.3 \mathrm{~g} / \mathrm{cm}^{3}$ to 0.21 for samples at $1.6 \mathrm{~g} / \mathrm{cm}^{3}$ density (Table 1 ), while the differences between slices within each subsample (Fig. 3) were not significant $(p=0.15)$.

There was a decline in the median pore size with bulk density (Table 1) but only the median pore diameter at a density of 1.6 was significantly different from those at 1.2 and 1.3 $(p<0.04)$. The changes in porosity and pore diameter show that when soils were packed at higher densities the overall pore volume declined and mainly the larger pores were reduced. However, for all samples the mean pore diameter remained an order of magnitude larger than a typical fungal diameter of 1-50 $\mu \mathrm{m}$ (Finlay, 2006). No significant differences were found for porosity and median pore diameter between replicated samples at this scale.

All subsamples had highly connected pore volumes with a minimum of $90 \%$ of the pore volume connected to a single large cluster for all densities. Figure 2 shows how the connected largest clusters and the remaining pore-space that did not belong to this cluster, is distributed throughout the soil sample. There were significant differences in connectivity between replicates at all densities $(p<0.001)$ indicating a greater variability of this parameter at this scale. In particular, the connectivity was significantly lower for the samples at higher densities $(95 \%$ for $1.5(p<0.015)$ and $90 \%$ for 1.6 , $(p<0.001))$ as compared to lower densities (97\% for 1.2, 1.3 and 1.4 soil bulk density).

\subsection{Effect of the physical characteristics on fungal invasion}

Due to the high connectivity of the pore space, the amount of biomass following fungal invasion displayed trends similar to those found for the porosity, with significant differences between treatments $(p<0.001)$ (Fig. 3b). As expected, biomass content decreased as porosity decreased, with the sample with the highest porosity also having the highest biomass after fungal invasion. The soil with the lowest porosity had an average biomass after fungal invasion of only $54 \%$ of that of the sample with the highest porosity (Fig. 3b, 4.13E5 for density 1.6 and 7.65E5 for density 1.3). This difference is comparable with the difference in 

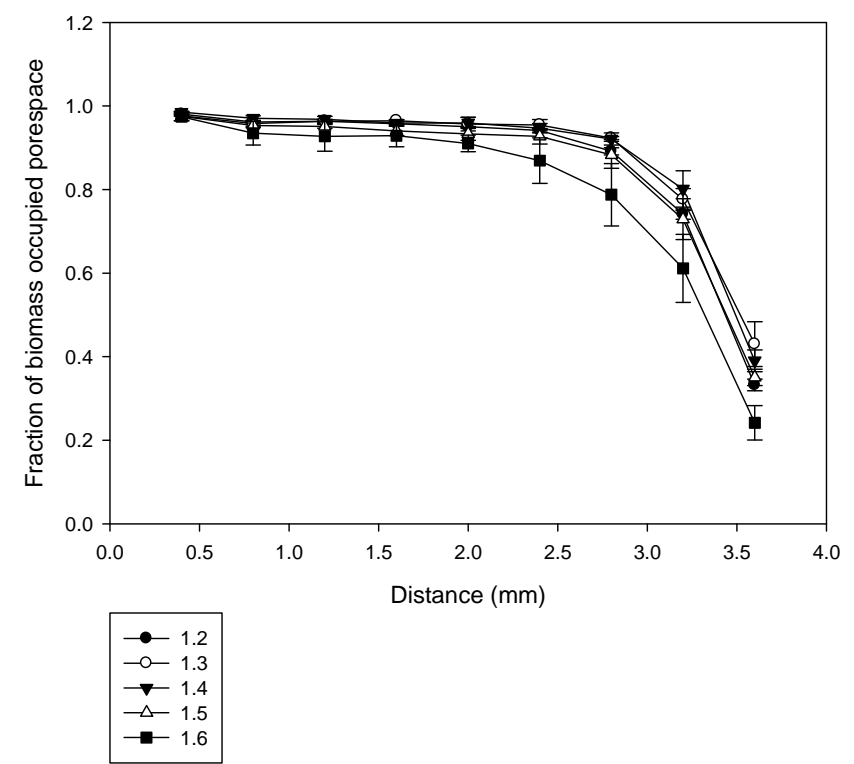

Fig. 4. Mean changes in the fraction of pore space occupied by biomass in each segment with distance from the site of inoculation, for soils at bulk-densities of $1.2,1.3,1.4,1.5$, and $1.6 \mathrm{~g} / \mathrm{cm}^{3}$.

the porosity which demonstrates the overriding importance of the total pore volume for fungal invasion. There was a noticeable drop $(p<0.001)$ in biomass content at distances further than approximately $2.5 \mathrm{~mm}$ from the site of inoculation (Fig. 3b). The drop in biomass content characterized the front of colony growth.

The amount of biomass per sample did not inform us about the spatial distribution of the biomass. To obtain a quantitative measure of the spatial colonization, we quantified the fraction of the pore space that became colonized. With increasing distance from the site of inoculation, the fraction of pores occupied by biomass declined steeply (Fig. 4). At distance $<2.5 \mathrm{~mm}$, nearly all of the pore space was occupied by fungal biomass. This reflects the high connectivity of the pore space for all samples. The drop in biomass at larger distances coincided with a drop in the fraction of pores that were occupied (Fig. 4). Soil packed at a density of $1.6 \mathrm{~g} / \mathrm{cm}^{3}$ (the most densely packed material) showed an earlier decline in the fraction of pores colonized with distance (Fig.4., $p<0.001$ ). As the porosity did not change with distance for these samples (Fig. 3a) it is most likely a consequence of the lower connectivity for this sample (Table 1). As a fungal colony spread into a soil sample, the larger pores were colonized first (as they were typically well connected, Fig. 5a) and this was followed by colonization of the smaller pores (Fig. 5b), and the invasion typically followed a sharp colony front (Fig. 5d and e).
(a) fungal growth, $t=8$
(b) fungal growth, $\mathrm{t}=12$
(c) soil structure

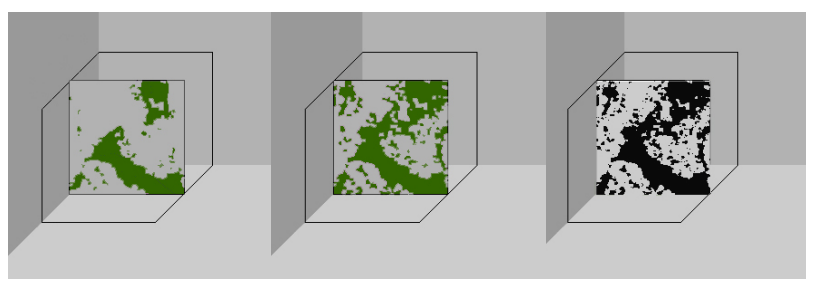

(d) ) fungal growth, $t=8$

(e) fungal growth, $t=12$

(f) soil structure

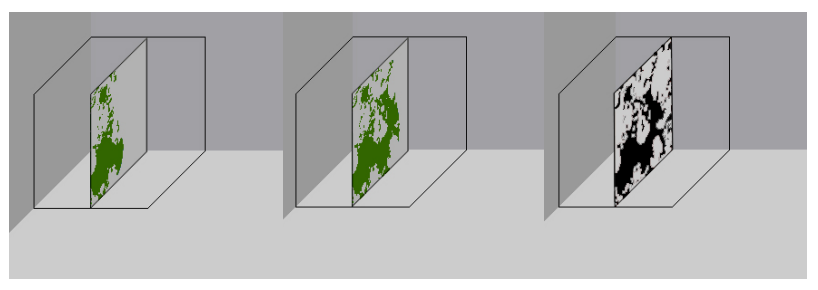

Fig. 5. 2-D slices through the $\mathrm{z}$ axis (a-c, perpendicular to the direction of spread) and $\mathrm{x}$ axis (d-f, in the direction of spread) showing biomass occupancy at $\mathrm{t}=8$ ( $\mathrm{a}, \mathrm{d}$, halfway the time required to spread through the entire volume), $t=12$ (b, e, at the time the colony had spread to the opposite side of the subsample), and corresponding pore space $(c, f)$ where solid - gray, pore space - black for a sample at a bulk density of $1.6 \mathrm{~g} / \mathrm{cm}^{3}$.

\subsection{Carbon level and dynamics of fungal invasion}

Differences in the dynamics of fungal invasion are shown in Fig. 6, which shows how in the middle of the sample the biomass increased with time. All treatments had a similar characteristic shape for the dynamics. Initially, for $\mathrm{t}<8$, the biomass was absent until the edge of a fungal colony had progressed sufficiently far into the soil sample. Once the edge of a colony reached a specified distance from the site of inoculation (here shown for $2.5 \mathrm{~mm}$ in Fig. 6) then the pore volume at that distance became rapidly colonized for all densities. However, the rate of colonization differed per treatment with the highest rates (sharpest increase) for the lower density samples. As expected, in a better connected sample (lower density soil) fungal biomass moved through the volume rapidly, almost as a steep front over time filling all available pore space. For the more densely packed soil, fungal invasion progressed slower (shown by the lower rate of increase) and the final level of fungal biomass was lower (Fig. 6).

The dynamics of fungal invasion were also determined by the availability of Carbon in the soil (Fig. 6b). Although the trends for fungal invasion were qualitatively similar for both resource levels, the limitation of carbon radically affected biomass content reducing the final level of biomass following invasion to $1 \%$. As expected, this reduction reflects the lower amount of $\mathrm{C}$ available for fungal growth. Limitation of $\mathrm{C}$ level also affected the rate of invasion. With unlimited resources colonization at a distance of $2.5 \mathrm{~mm}$ from the site 

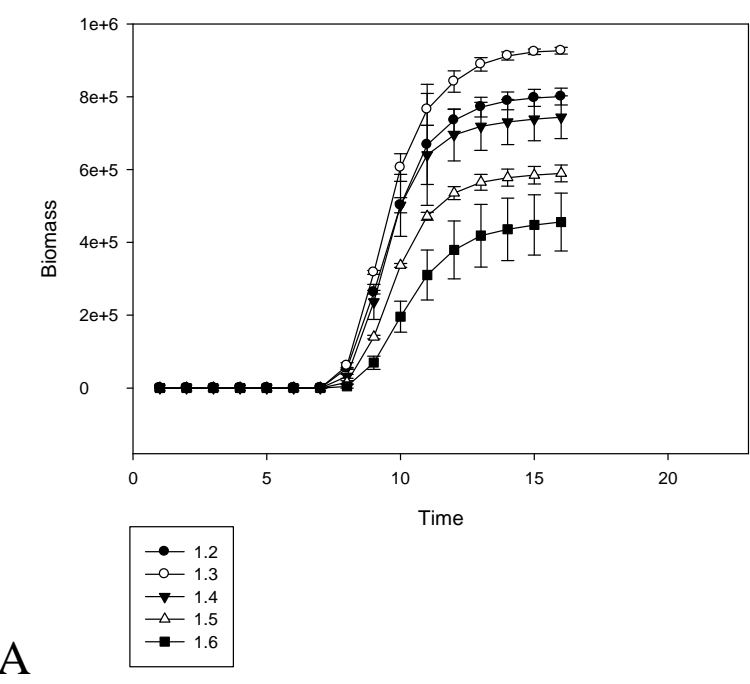

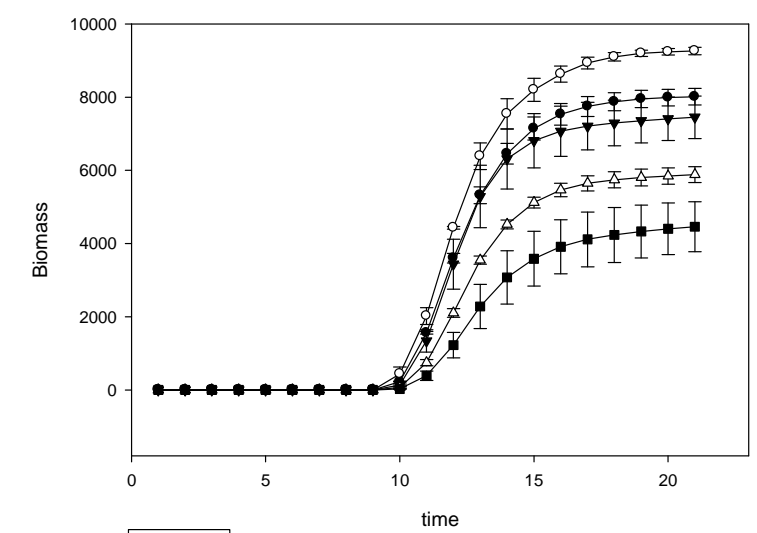

B

Fig. 6. Mean dynamics of biomass at the middle of each subsample (5th segment) with "unlimited" (A) and limited (B) resource, for soils at bulk-densities of $1.2,1.3,1.4,1.5$, and $1.6 \mathrm{~g} / \mathrm{cm}^{3}$. Note that the scales for the amount of biomass differ for both resource levels.

of inoculation started at $\mathrm{t}=7$, but the starting point for microcosms with limited resources was delayed to $t=10$. Overall it also took longer for soils with a lower resource level for the fungal colony to spread through the entire soil volume. At high resource levels this took on average (17 time units), whereas at lower resource levels this increased to 22 time units.

\section{Discussion}

One of the difficulties in studying fungal invasion is the lack of suitable quantitative techniques that enable monitoring of fungal spread through soil over time. Techniques used so far include plating out of aggregates to obtain colony forming units, ergosterol essay (Feeney et al., 2006), MAb-ELISA for specific species (Otten et al., 1997) or quantification by PCR (Lopez-Mondejar et al., 2009). These techniques however only enable a single snap shot in time, and, perhaps more importantly, require the destruction of the physical environment which contributed to the growth dynamics. Hence we obtain quantitative information of fungal biomass in bulksoil samples, but no information about the spatial location of the fungi within the soil environment at the microscopic scales where interactions and processes occur. To date, the only way by which quantitative information about the spatial distribution of fungal mycelium in undisturbed soil samples can be obtained, is in biological thin sections (Tippkotter and Ritz, 1996), but even there the information is essentially constrained to a 2-D plane from the 3-D soil environment. It is therefore important to develop a novel method for analysis and visualisation of the effect of the heterogeneity of the pore volume on microbial processes in 3-D.
The simulations showed that for all soil samples a large percentage of the pore space was colonized by fungi. This is an inherent property of the model, which describes the fungal invasion as a diffusive process, as a result of which all connected pore space would be expected to be colonized eventually. As a result, for a well connected pore volume, the total porosity is the key determinant of the density of fungal biomass following invasion. Close to the site of inoculation, nearly all pore space became colonized for all treatments (Fig. 4). However, if the connectivity is less than 1 (e.g. with increasing bulk density), then progressively less pore space becomes colonized at distances further away from the site of inoculation as biomass spreads only through a connected network. In that case the connectivity of the pore space becomes an increasingly important factor.

In accordance with experimental data for the invasive spread of R. solani in the same soil samples (Harris et al., 2003) pore volumes with a larger diameter became colonized first. Behind the progressing colony front in the larger pores, the fungi subsequently colonize the pore volumes with smaller diameters. However, the model does appear to overestimate the colonization of smaller pores as experimental data showed that there was a greater preference for larger connected pores (Otten et al., 2004). This could be the result of a more heterogeneous distribution of Carbon in the soil compared to the simulations, or a result of blockage of pores by water, which means that the connectivity of the pore volume in the simulations is overestimated as we assume all pores to be filled with air. Future work may need to address this in more detail where we can consider extending the modelling approach by including mechanisms that enhance spread through larger pores. 
On nutrient rich agar plates, fungi typically form circular colonies, with a step change in biomass density at the advancing colony edge. In nutrient poor systems, colony spread is often more heterogeneous as fungi switch from an exploitative to an explorative mode (Boddy et al., 2009). In soil, we similarly expect this colony shape to be mediated by the heterogeneity of the pore volume with the advancing edge of a colony less clearly defined as the colony needs to negotiate a tortuous pathway of connected pores, resulting in a more gradual change in biomass density towards the growing edge of the colony. For all treatments, we observed nevertheless steep declines in biomass density and in the percentage of colonized pores at the colony growing front. For loosely packed soil the change in density at the front was steeper, characteristic of faster more homogeneous growth (Figs. 3, 4). Fungi spreading though soil packed at higher densities had a smoother decay in density at the growing front. At these densities soil had a smaller amount of available pore space, which was less connected and had smaller median pore size (Table 1). This trend in the effect of pore geometry on fungal colony development is in agreement with experimental results reported by Harris (Harris et al., 2004) and Ritz (Ritz and Young, 2004) where it was shown that fungi spread faster through large pores with a high percentage of air-filled spaces. Fungi in volumes with small, poorly connected pores grow slower but colonies tend to have denser biomass (Harris, 2003; Ritz, 2004). While the differences may appear to be small, it should be noted that such small differences can have a significant impact on larger scale invasive spread of fungi and can make a fungal species switch from invasive to non-invasive spread (Bailey et al., 2000; Kleczkowski et al., 1997).

Current CT tomography systems have a number of limitations. One of the biggest challenges is the ratio between sample size and resolution (voxel size), with a smaller resolution for larger sample sizes. With our system, the maximum size of the sample which can be scanned is $25 \times 20 \mathrm{~cm}$, at a resolution of approximately $150 \mu \mathrm{m}$. The disadvantage of a lower resolution is that we loose information about micropores, and as a result can loose the connectivity in the pore network. Whereas with our system we could obtain resolutions $(5 \mu \mathrm{m})$ smaller than typical fungal diameters, this would have required us to restrict the sample sizes to be too small to be meaningful for fungal colonies and the length scales at which heterogeneity in physical conditions is observed in these samples. The sample size we used in this paper is still small compared to sizes that ordinarily would be assumed representative for a field. However, the small sizes were representative for the relatively homogeneous samples used in this study, as they were obtained by repacking sieved soil. In our statistical analysis of the quantification of pore space, we allowed for variability within treatments (between subsamples), as well as between treatments, and we showed that the difference between sub-samples within a treatment was not significant, but that difference between sub-samples from different treatments was significant. It is however not possible to extrapolate this result to soil samples from natural fields, and no extrapolation can be made towards the effect of management on fungal growth from these small samples. Young and Ritz (1999) reviewed the impact of tillage on colonies of microbes and argued that typical soil disturbance may not be significant for fungal colonies at small scales. The results in this study should be seen as a first step towards understanding the effect of soil management on fungal growth. Although the resolution of approximately $30 \mu \mathrm{m}$ in the scans used in this paper is larger than a typical hyphal diameter, it is smaller than typical internodes' length, and appropriate for fungal colonization which is typically determined by the ability of fungal hyphae to branch within a confined space (Otten and Gilligan, 1998). In addition, the resolution is identical to the approximate resolutions of thin sections, the only technique currently available to visualize fungi in soil, enabling a qualitative comparison with experimental data.

Another bias in the results can be caused by thresholding, which is one of the most crucial steps in image processing where an operator differentiates between solid material and pore-space. A single threshold value as used in this paper is known to overestimate large pores and underestimate small pores and thin valleys. However, it was also shown that for the type of samples in this study, a reasonable agreement with the overall porosity was found (Baveye et al., 2010). Tarquis et al. $(2008 ; 2009)$ showed the impact of thresholding on various geometrical descriptors of pore geometry, but the consequences for soil functioning is still largely unknown, which can be explored with the modelling framework presented in this paper.

\section{Conclusions}

In this paper we showed how a combination of X-ray tomograpghy (to characterise the soil structure) and mathematical modelling (to model fungal invasion in 3-D structural heterogeneous environments) can be used to identify the effect of pore geometry on fungal spread. Such a analysis is an essential first step towards a theoretical basis for management decision taking that would aim to maintain or support biodiversity in soils, which is currently lacking. The theoretical approach enables separation of nutritional and structural effects on fungal growth, which is difficult to achieve by other means. We showed that both the dynamics and the degree of colonization are not just affected by the porosity, but also depend on the connectivity of the pore volume. Further work is now required to identify how colonization efficacy relates to fungal traits and heterogeneity in the availability of carbon. 
Acknowledgements. The authors wish the acknowledge support for this study from the University of Abertay Dundee and from the Scottish Alliance for Geosciences, Environment and Society (SAGES). The University of Abertay Dundee is a charity registered in Scotland, no: SC016040. We also thank D. Grinev for discussions on the use of X-ray CT for quantifying soil structure.

Edited by: A. M. Tarquis

\section{References}

Bailey, D. J., Otten, W., and Gilligan, C. A.: Saprotrophic invasion by the soil-borne fungal plant pathogen Rhizoctonia solani and percolation thresholds, New Phytologist, 146, 535-544, 2000.

Baveye, P. C., Laba, M., Otten, W., Bouckaert, L., Sterpaio, P. D., Goswami, R. R., Grinev, D., Houston, A., Hu, Y., Liu, J., Mooney, S., Pajor, R., Sleutel, S., Tarquis, A., Wang, W., Wei, Q., and Sezgin, M.: Observer - dependent variability of the thresholding step in the quantitative analysis of soil images and X-ray microtomography data, Geoderma, 157, 51-63, 2010.

Boddy, L., Hynes, J., Bebber, D. P., and Fricker, M. D.: Saprotrophic cord systems: dispersal mechanisms in space and time, Mycoscience, 50, 9-19, doi:10.1007/s10267-008-0450-4, 2009.

Bossuyt, H., Denef, K., Six, J., Frey, S. D., Merckx, R., and Paustian, K.: Influence of microbial populations and residue quality on aggregate stability, Appl. Soil Ecol., 16, 195-208, 2001.

Boswell, G. P., Jacobs, H., Davidson, F. A., Gadd, G. M., and Ritz, K.: Functional consequences of nutrient translocation in mycelial fungi, J. Theor. Biol., 217, 459-477, doi:10.1006/yjtbi.3048, 2002.

Dane, J. H., Hopmans, J. W., Romano, N., Nimmo, J., and Winfield, K. A.: Soil water retention and storage - Introduction, in: Methods of Soil Analysis, Part 4, Physical Methods,, edited by: Dane, J. H., Soil Sci. Soc. Am., 2002.

Deurer, M., Grinev, D., Young, I., Clothier, B. E., and Mueller, K.: The impact of soil carbon management on soil macropore structure: a comparison of two apple orchard systems in New Zealand, Eur. J. Soil Sci., 60, 945-955, doi:10.1111/j.13652389.2009.01164.x, 2009.

Edelstein, L.: The Propagation of Fungal Colonies - a Model for Tissue-Growth, J. Theor. Biol., 98, 679-701, 1982.

Edelstein, L. and Segel, L. A.: Growth and Metabolism in Mycelial Fungi, J. Theor. Biol., 104, 187-210, 1983.

Falconer, R. E., Bown, J. L., White, N. A., and Crawford, J. W.: Biomass recycling and the origin of phenotype in fungal mycelia, P. Roy. Soc. B-Biol. Sci., 272, 1727-1734, doi:10.1098/rspb.2005.3150, 2005.

Falconer, R. E., Bown, J. L., White, N. A., and Crawford, J. W.: Biomass recycling: a key to efficient foraging, Oikos, 9, 2007.

Falconer, R. E., Bown, J. L., White, N. A., and Crawford, J. W.: Fungal Interactions in Fungi, The Royal Society Interface, 5, 2008.

Feeney, D. S., Bengough, A. G., Hallett, P. D., Rodger, S., White, N., and Young, I. M.: Assessing the impact of biological exudates associated with soil water repellency, Adv. Geoecol., 38, 475-483, 2006.

Finlay, R. D.: The fungi in Soil, in: Modern soil microbiology., edited by: Van Elsas, J., Jansson, J. K., and Trevors, J. T., CRC
Press, 2006.

Hardin, J. W. and Hilbe, J. M.: Generalized Estimating Equations, Stata Corporation, 2003.

Harris, K., Young, I. M., Gilligan, C. A., Otten, W., and Ritz, K.: Effect of bulk density on the spatial organisation of the fungus Rhizoctonia solani in soil, FEMS Microb. Ecol., 44, 45-56, 2003.

Kleczkowski, A., Gilligan, C. A., and Bailey, D. J.: Scaling and spatial dynamics in plant-pathogen systems: From individuals to populations, P. Roy. Soc. Lond. B. Bio., 264, 979-984, 1997.

Lark, R. M.: Exploring scale-dependent correlation of soil properties by nested sampling, Eur. J. Soil Sci., 56, 307-317, doi:10.1111/j.1365-2389.2004.00672.x, 2005.

Lopez-Mondejar, R., Anton, A., Raidl, S., Ros, M., and Pascual, J. A.: Quantification of the biocontrol agent Trichoderma harzianum with real-time TaqMan PCR and its potential extrapolation to the hyphal biomass, Bioresource Technol., 101, 28882891, 2009.

Meskauskas, A., Fricker, M. D., and Moore, D.: Simulating colonial growth of fungi with the Neighbour-Sensing model of hyphal growth, Mycol. Res., 108, 1241-1256, doi:10.1017/s0953756204001261, 2004.

Nunan, N., Ritz, K., Crabb, D., Harris, K., Wu, K. J., Crawford, J. W., and Young, I. M.: Quantification of the in situ distribution of soil bacteria by large-scale imaging of thin sections of undisturbed soil, Fems Microbiol. Ecol., 37, 67-77, 2001.

Or, D., Smets, B. F., Wraith, J. M., Dechesne, A., and Friedman, S. P.: Physical constraints affecting microbial habitats and activity in unsaturated porous media - a review., Adv. Water Resour., 30, 2007.

Otten, W. and Gilligan, C. A.: Effect of physical conditions on the spatial and temporal dynamics of the soil-borne fungal pathogen Rhizoctonia solani, New Phytologist, 138, 629-637, 1998.

Otten, W., Gilligan, C. A., and Thornton, C. R.: Quantification of fungal antigens in soil with a monoclonal antibody-based ELISA: analysis and reduction of soil-specific bias, Phytopathology, 730-736, 1997.

Otten, W., Harris, K., Young, I. M., Ritz, K., and Gilligan, C. A.: Preferential spread of the pathogenic fungus Rhizoctonia solani through structured soil, Soil Biol. Biochem., 36, 203-210, 2004.

Ritz, K. and Young, I. M.: Interactions between soil structure and fungi, Mycologist, 18, 52-59, 2004.

Stacey, A. J., Truscott, J. E., and Gilligan, C. A.: Soil-borne fungal pathogens: scaling-up from hyphal to colony behaviour and the probability of disease transmission, New Phytol., 150, 169-177, 2001.

Tarquis, A. M., Heck, R. J., Andina, D., Alvarez, A., and Anton, J. M.: Pore network complexity and thresholding of 3-D soil images. Ecological complexity 6, 230-239, 2009.

Tarquis, A. M., Heck, R. J., Grau, J. B., Fabregat, J., Sanchez, M. E., and Anton, J. M.: Influence of thresholding in mass and entropy dimension of 3-D soil images, Nonlinear Proc. Geoph., 15, 881891, 2008.

Taylor, L. L., Leake, J. R., Quirk, J., Hardy, K., Banwart, S. A., and Beerling, D. J.: Biological weathering and the long-term carbon cycle: integrating mycorrhizal evolution and function into the current paradigm, Geobiology, 7, 171-191, doi:10.1111/j.14724669.2009.00194.x, 2009.

Tippkotter, R. and Ritz, K.: Evaluation of polyester, epoxy and acrylic resins for suitability in preparation of soil thin sections 
for in situ biological studies, Geoderma, 69, 31-57, 1996.

Tisdall, J. M.: Fungal Hyphae and Structural Stability of Soil, Aust. J. Soil Res., 29, 729-743, 1991.
Young, I. M. and Crawford, J. W.: Interactions and self-organization in the soil-microbe complex, Science, 304, 1634-1637, 2004

Young, I. M. and Ritz, K.: Tillage, habitat space and function of soil microbes, Soil \& Tillage Research, 53, 201-213, 2000. 